\title{
Preparation and Properties of Novel Microporous Hybrid Hydrogels with Poly(ethylene glycol) Dimethacrylate In situ Polymerization
}

\author{
Wang Pei \\ Department of Materials Science and Engineering \\ Dalian Maritime University \\ Dalian, China \\ peterwp@126.com
}

Wang Shuai

Department of Respiratory Medicine

First Affiliated Hospital of Dalian Medical University Dalian, China

Wangshuai501@126.com

\author{
Li Liang \\ Department of Materials Science and Engineering \\ Dalian Maritime University \\ Dalian, China \\ liliang2013@126.com \\ Liu Lian \\ Department of Materials Science and Engineering \\ Dalian Maritime University \\ Dalian, China \\ lian1iu2013@163.com
}

\begin{abstract}
Hydrogels have been widely used for biomedical and tissue engineering applications, and there are a lot of both synthetic and natural systems used for these purposes. In this study, we described the synthesis, characterization and properties of a novel hybrid PEG-based hydrogel in situ polymerization. The hybrid hydrogel was prepared by freeradical redox polymerization using TEMED/APS as initiators and $\mathbf{N}, N^{\prime}-$ methylenebisacrylamide (BIS) as crosslinker at $60{ }^{\circ} \mathrm{C}$. The physicochemical properties of hybrid hydrogel were characterized by FT IR spectroscopy and scanning electron microscopy (SEM), while swelling kinetics and gel content were calculated. The results indicated swelling degree in distilled water could come to a maximum of $537 \%$ with $0.3 \mathrm{wt} \%$ CNTs. SEM images showed the micron pore size of hydrogels could be adjusted by changing the cross-linker mass from 2 wt\% to 10 wt \%. So, the obtained hydrogels with appropriate crosslinkage would be exploited as biomedical applications, such as tissue engineering and three dimensional tissue scaffolds.
\end{abstract}

Keywords- Poly(ethylene glycol); hybrid hydrogel; in situ polymerization style; carboxylated carbon nanotubes; physicochemical property

\section{INTRODUCTION}

Poly(ethylene glycol) (PEG) hydrogels is one of the widely studied and used hydrophilic polymer in the biomaterials field[1-3]. The pioneering research showed that synthetic hydrogels is a promising class of biomaterials since they can be easily to produce desirable mechanical and chemical properties for both in vitro and in vivo biological and medical applications, such as control drug delivery and cell scaffolds for tissue engineering[4,5]. In particular, in situ formed hydrogels[6,] have recently been recognized for their potential biomedical applications. Making a sol-to-gel transition mechanism could provide a simple implantation method by injecting an aqueous solution (sol)[7,8] with pharmaceutical agents or cells into a target site to form a hydrogel depot, without surgical procedures or complicate fabrication processes.

Above mentioned field, poly(ethylene glycol) (PEG) hydrogel[9] is one of the most widely studied and used materials since PEG hydrogel are nontoxic, nonimmunogenic, and approved by the US Food and Drug Administration (FDA) for many clinical uses. Acrylatefunctionalized PEG monomers would be easy to form a crosslinked macromers by redox- or photopolymerization. Despite the widespread use of PEG-based hydrogel biomaterials, it lacks the desired mechanical properties and is limited to further medical engineer applications. Inorganic-organic hybrid materials with carbon nanotubes(CNTs) [10,11] has aroused interest for researcher to enhanc the mechanical properties of materials. Hybrid hydrogels can overcome the disadvantages of hydrogels by combining the advantages of the components.

A significant challenge to cross-linking hydrogels is to control the shape and size of microparticle and microstructure. However, it was more or less limited by the use of previously reported techniques [12], such as, emulsification, microfluidics, and shear-induced droplet formation [13]. So, in this study, firstly, we synthesized a series of copolymer hydrogels with poly(ethylene glycol) dimethacrylate (PEGDMA) (200 g/mol), 2-hydroxyethyl methacrylate (HEMA) by a effective free-radical redox polymerization method. The influence of different amounts of cross-linker N, N'-methylene bisacrylamide (BIS) and HEMA on the physical properties and surface morphology were discussed. Moreover, inorganic-organic hybrid materials are explored as the reinforcing filler. 


\section{EXPERIMENTAL}

\section{A. Synthesis}

PEGDMA-co-HEMA hydrogel series were prepared in solution precipitation polymerization. For example, a solution of $0.02 \mathrm{~mol}$ poly(ethylene glycol) dimethacrylate (PEGDMA, 200g/mol, purity $>99$ wt $\%$ ), $0.012 \mathrm{~mol} \mathrm{2-}$ hydroxyethyl methacrylate (HEMA, purity >95 wt \%), $0.001 \mathrm{~mol} \mathrm{~N}, \mathrm{~N}$-methylenebisacrylamide (BIS, > $98 \mathrm{wt} \%$ ), and $0.040 \mathrm{~g}$ of carbon nanotubes(CNTs) was mixtured by ultrasonic dispersion for $10 \mathrm{~min}$ in distilled water. The polymerization were operated for $40 \sim 60 \mathrm{~min}$ at $60{ }^{\circ} \mathrm{C}$ after adding $0.35 \mathrm{mmol}$ APS and $8 \mathrm{mmol}$ TEMED with $\mathrm{N}_{2}$ atmosphere. Then, the hydrogels were immersed in anhydrous alcohol overnight to remove unreacted monomer. Samples were vacuum freeze-drying for $48 \mathrm{~h}$ before characterization or measurement. The structure of cross-linked copolymers was shown in Fig .1.

\section{B. Characterizations and Measurement}

Infrared absorption spectra were recorded by attenuated total reflectance infrared spectroscopy (ATR-IR) with a Nicolet 20DXB (American) infrared spectrometer in the range of $4000-500 \mathrm{~cm}^{-1}$ range. Scanning electron microscopy (SEM) (SUPRA 55 SAPPHIRE, ZEISS) was used to characterize the surface and cross-section morphologies of hydrogels. The cross-section was fractured in liquid nitrogen. A thin gold layer was coated on the surface.

Swelling degree $(S)$ was calculated according to the formula: $\quad S=m_{s}-m_{d} / m_{s}$

wherein, the dry mass $m_{\mathrm{d}}$ was recorded after drying under ambient conditions for $24 \mathrm{~h}$ and then in vacuum for $12 \mathrm{~h}$; ms was measured after samples was immersed in distilled water at an interval time and taken out of solution for a quickly dried with a tissue paper.

Gel content: A certain quality of the dried gel $\left(M_{0}\right)$ was soaked into chloroform for $48 \mathrm{~h}$ and leached by anhydrous alcohol and dried in vacuum at $60{ }^{\circ} \mathrm{C}$ for $24 \mathrm{~h}$. Then, gel samples were weighted as $M_{1}$. All samples were tested three times. Gel mass fraction was recorded as: $G=M_{1}$

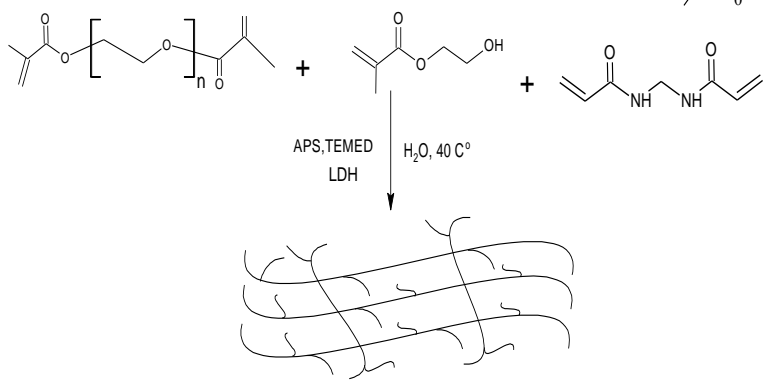

Figure 1. Schematic drawings of hydrogel PEGDMA-co-HEMA.

\section{RESULTS AND DISCUSSION}

\section{A. Hydrogel characteristics}

In considering the biological applications of a hydrogel, the gel forming reaction is recommended to occur in water, wherein the bioactive agents or cells are dissolved or suspended. Free radicals produced in water by redox- reactions can be used for polymerization of methacrylate functionalized macromers to prepare a hydrogel using TEMED/APS as a redox-initiating system. All the samples were prepared at a lower temperature of $60{ }^{\circ} \mathrm{C}$ and only for 40 min under vacuum freeze-drying for keeping the natural cross-linking structure instead of dried under high temperature.

From the Fig .2, we can observe the main peaks of PEGDMA-co-HEMA hydrogels. The broad absorption band at about $3441 \mathrm{~cm}^{-1}$ would be ascribed to the $-\mathrm{OH}$ group stretching for HEMA, The bands at $2886 \mathrm{~cm}^{-1}$ resulted from the asymmetric and symmetric stretching modes of $\mathrm{CH}_{2}$ groups. PEGDMA showed characteristic peaks at $1730 \mathrm{~cm}^{-1}(\mathrm{C}=\mathrm{O}$ stretch $), 1485-1445 \mathrm{~cm}^{-1}(\mathrm{CH}$ asymmetric deformation) and $1106 \mathrm{~cm}^{-1}(\mathrm{C}-\mathrm{O}-\mathrm{C}$ asymmetric stretch). The results confirmed the structure of PEGDMA-co-HEMA.

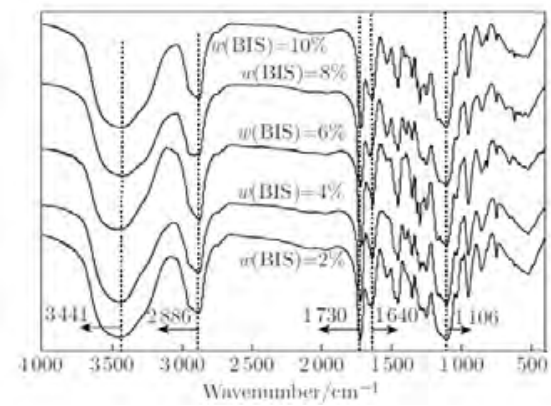

Figure 2. FTIR spectra of PEGDMA-co-HEMA with different BIS dosage

\section{B. Hydrogels swelling properties}

Swelling properties played a great role in the hydrogels application. In thermodynamic view, the extent of hydrogels swelling is determined by a balance between enthalpic gain (as a result of solution of the polymer network by water) and entropic loss, when Gaussian chain segments stretch during swelling and lose possible conformations.

We measured the swelling kinetic with different content cross-linker to illustrate the absorbing speed at intervals. Kinetic data were shown in Fig. 3 and Fig.4 with different monomer mass from $0 \mathrm{wt} \%$ to $20 \mathrm{wt} \%$ and different BIS content from $2 \mathrm{wt} \%$ to $10 \mathrm{wt} \%$ in distilled water. As we can be seen from the results, the absorption speed of gels had a rapid increase within the beginning 50 minutes. As a further extension time, absorption rate increased slowly and reached to a basically swelling balance up to $450 \mathrm{~min}$. In equilibrium, from Fig.3, hydrogels with 20\% HEMA and 6\% crosslinker showed higher water absorption degree, the maximum value of 135\%. In Fig.4, PEGDMA-co-HEMA with $6 \mathrm{wt} \%$ BIS showed higher swelling degree in equilibrium, but decreased only to $33 \%$ with BIS content down to $2 \mathrm{wt} \%$. It could be explained that the size of hydrogel cavities mainly depend on crosslinking density according to the content of the second monomer and crosslinker. The different aperture and the crosslinking density had great influences on the ability of water combined into the molecular chain.

We further investigated the gel weight content of the samples to illustrate the swelling ability by changing the 
BIS mass. Obviously, in Fig.5, the samples exhibited a higher degree of crosslinking (more than $88 \mathrm{wt} \%$ gel) as crosslinker was added up to $6 \mathrm{wt} \%$. The gel data were consistent with the results of the swelling.

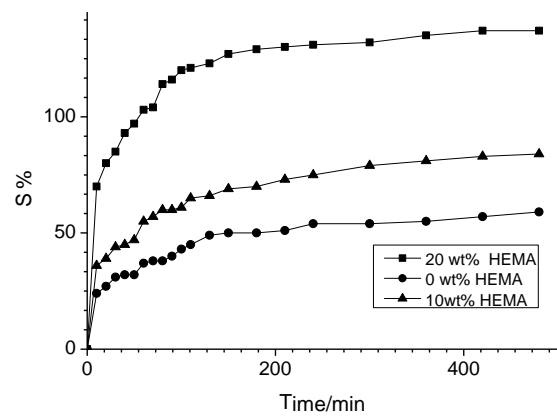

Figure 3. Swelling and kinetic data of PEGDMA-co-HEMA with different HEMA amounts

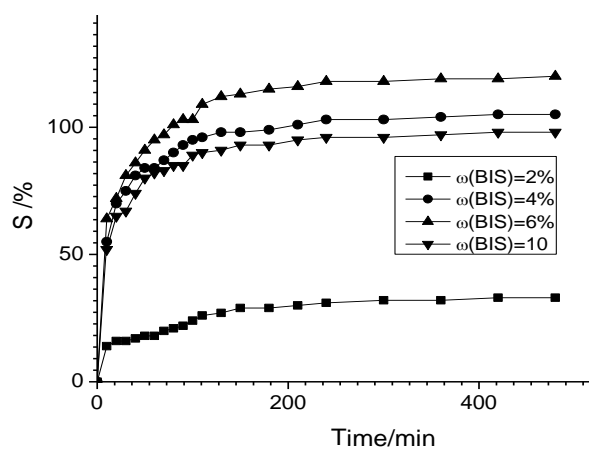

Figure 4. Swelling and kinetic data of PEGDMA-co-HEMA with different BIS amounts

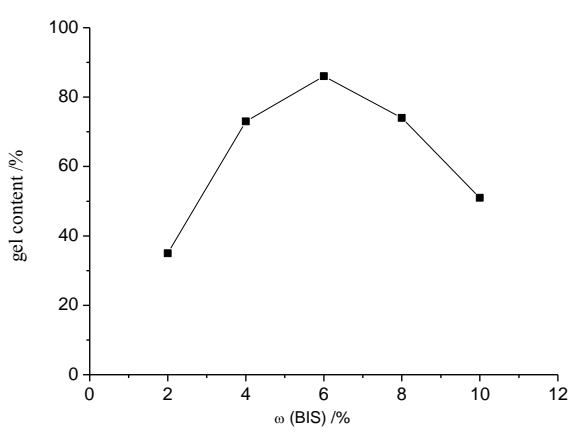

Figure 5. Gel mass fraction of hydrogels(10\% of HEMA) with different crosslinker amount

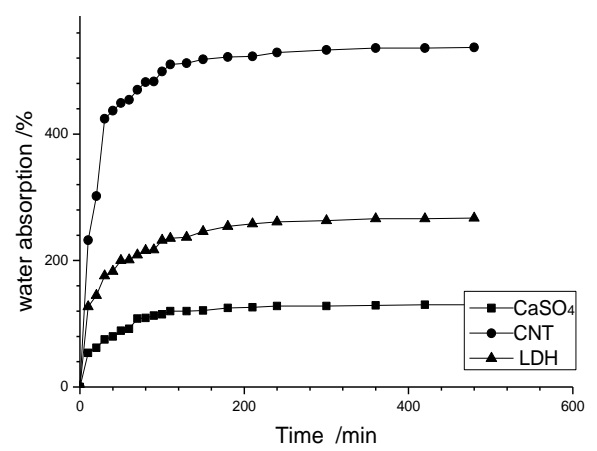

Figure 6. Water absorption of PEGDMA $(10 \%$ of HEMA) with different nanoparticles
It should be pointed out that the hydrogels with nanofiller including calcium sulfate whisker $\left(\mathrm{CaSO}_{4}\right)$, carbon nanotubes(CNTs) and layered double hydroxides (LDHs), had a much higher water absorption rate. In particular, the equilibrium value was as high as $537 \%$ with $0.3 \mathrm{wt} \%$ CNTs in Fig.6. So, the addition of CNTs would have a significant impact not only to the polymerization process and further to the pore structure of the hydrogel. The results in our studies also showed the regularity. In the next work, we would do further research to explore the change of crosslink structure in this system.

\section{Morphology Analysiss}

The internal structure of microscale hydrogel was related to cross-linking mesh. To date, this had not been a simple method to controlling the shape and size of microparticles and microstructures. We hoped that it could be improved by changing the amount of cross-linker. So, the image of SEM, shown in Fig.7, was taken from the fractured surface. It could be seen that the figures exhibited uniform microparticle morphology with micron pore size. The microparticle morphology would have obviously difference by changing the amount of crosslingker and nanofillers. The results showed that CNTs addition resulted in tighter crosslink density and pore size became smaller.

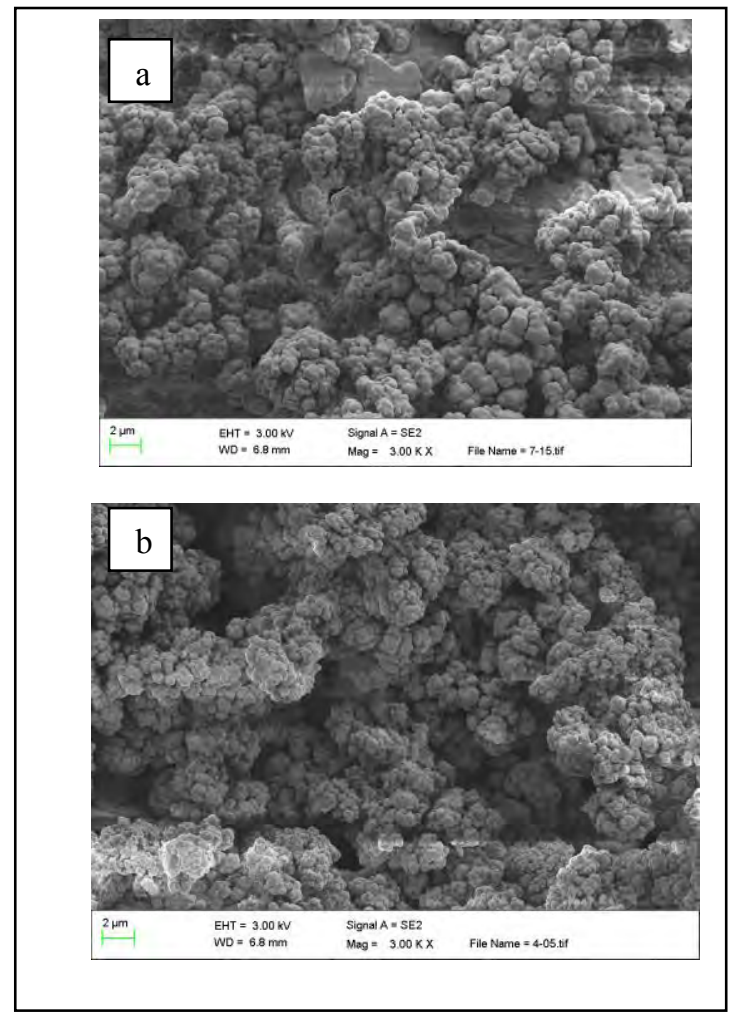




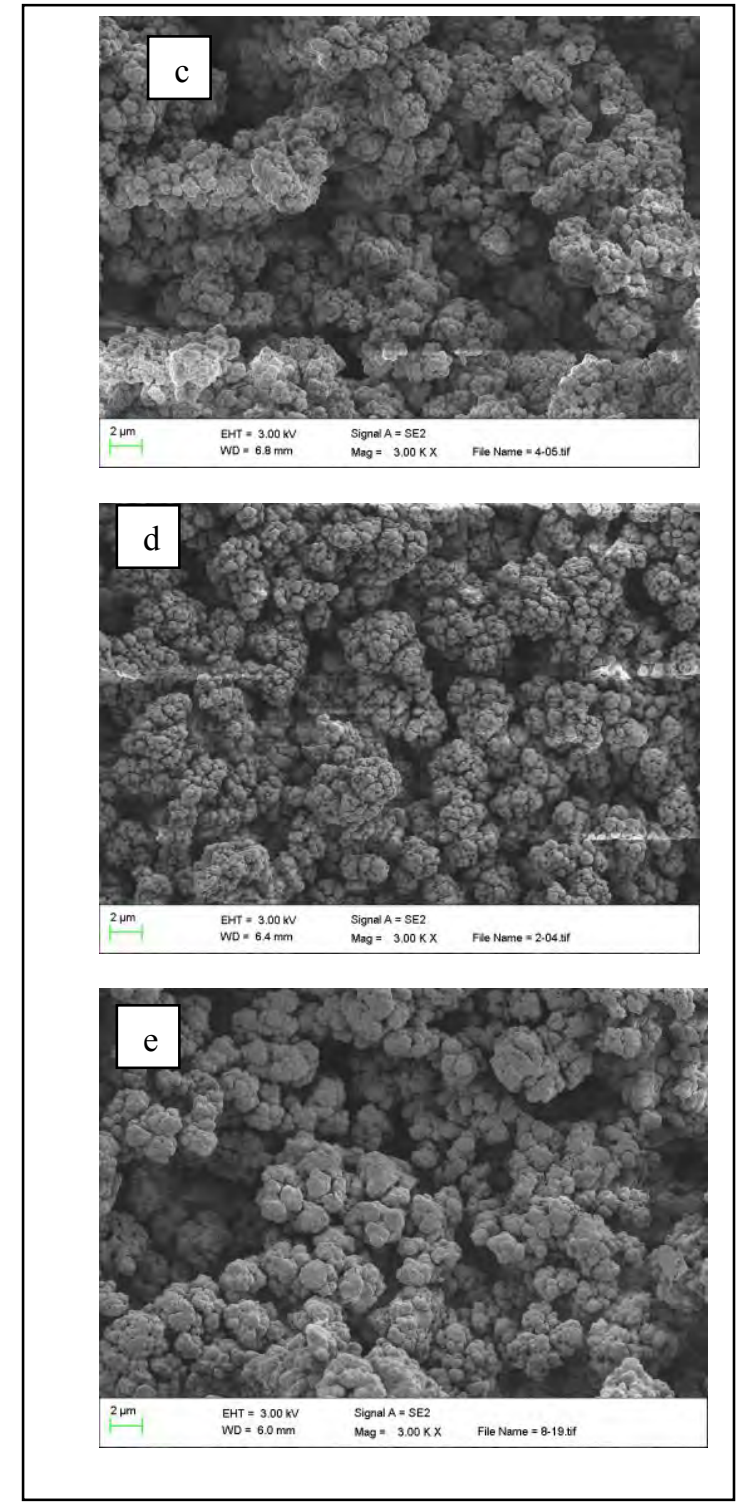

Figure 7. SEM images of PEGDMA-co-HEMA fracture surface with a)PEGDMA-20\%HEMA- $4 \%$ BIS; $\quad$ b)PEGDMA-20\%HEMA- $8 \%$ BIS; c)PEGDMA- $20 \%$ HEMA- $8 \%$ BIS- $0.5 \% \mathrm{CaSO}_{4}$; d)PEGDMA- $0 \%$ HEMA$8 \%$ BIS-0.5\% LDHs; e)PEGDMA- $0 \%$ HEMA- $8 \%$ BIS- $0.5 \%$ CNTs

\section{CONCLUSION}

In conclusion, a novel hybrid hydrogel of PEGDMAco-HEMA/CNTs were prepared in situ polymerization using a simple method to controlling the shape and size of microparticles and microstructures. Hydrogels exhibited highly-swollen behavior and swelling ratio. SEM images showed the micron pore size of inner hydrogels could be adjusted within the range of several micrometers by changing the cross-linker or nanofillers content. CNTs were randomly dispersed to the hydrogel by ultrasonic dispersion and the physicochemical properties would be improved. These could be designed to exploit as biomedical applications, such as tissue engineering and drug delivery.

\section{ACKNOWLEDGMENT}

This work was supported by the Natural Science Foundation of Liaoning Province (2014025011) and Fundamental Research Funds for the Central Universities (3132014076, 3132014323).

\section{REFERENCES}

[1] S. Rimmer, Biomedical Hydrogels, 1st ed.,Cambridge:Woodhead, 2011, pp.228-255.

[2] Q. Yang, N. Adrus, F. Tomicki and M. Ulbricht - Eomposites of Functional Polymeric Hydrogels and Porous Membranes," J Mater. Chem., Vol. 21, Nov. 2011, pp. 2783-2811, doi:10.1039/C0JM02234A.

[3] T. Ekblad, G. Bergstroem and T. Ederth, et al., Poly(ethylene glycol)-Containing Hydrogel Surfaces for Antifouling Applications in Marine and Freshwater," Biomacromolecules, Vol.9, Aug. 2008, pp.2775-278, doi: $10.1021 / \mathrm{bm} 800547 \mathrm{~m}$.

[4] N. A. Peppas, J. Z. Hilt, A. Khademhosseini and R. Langer, Hydrogels in Biology and Medicine: From Molecular Principles to Bionanotechnology," Advanced Materials, Vol.18, May 2006, pp.1345-1360, doi: 10.1002/adma.200501612.

[5] A. S. Hoffman, Hydrogels for Biomedical Applications," Adv Drug Deliv. Rev., Vol.54, Jan. 2002, pp.3-12, doi: 10.1016/S0169409X(01)00239-3.

[6] H. J. Chung and T. G. Park, Self-assembled and Nanostructured Hydrogels for Drug Delivery and Tissue Engineering," Nano Today, Vol. 4, Oct. 2009, pp. 429-437, doi:10.1016/j.nantod. 2009.08.008.

[7] M. H. Park, M. K. Joo, B.G. Choi and B. Jeong, Biodegradable Thermogels," Acc. Chem. Res., Vol.45, Oct, 2011, pp 424-433, doi:10.1021/ar200162j.

[8] Y, Li, J. Rodrigues and H. Tomás, fnjectable and biodegradable hydrogels: gelation, biodegradation and biomedical applications," Chem. Soc. Rev., Vol. 41, Nov. 2011, pp, 2193-221, doi 10.1039/C1CS15203C.

[9] J. B. Leach and C. E. Schmidt, -Characterization of protein release from photocrosslinkable hyaluronic acidpolyethylene glycol hydrogel tissue engineering scaffolds", Biomaterials, Vol.26, Jan. 2005, pp.125-135, doi:10.1016/j.biomaterials.2004.02.018.

[10] N.W. Kam, M. O'Connell, J.A. Wisdom, H. Dai, Earbon Nanotubes as Multifunctional Biological Transporters and Nearinfrared Agents for Selective Cancer Cell Destruction", Proc. Natl. Acad. Sci. USA, pp. 11600-11605, Aug. 2005. doi:10.1073 /pnas.0502680102

[11] M. Prato, K. Kostarelos and A. Bianco, Functionalized carbon nanotubes in drug design and discovery,” Acc. Chem. Res., Vol.41, Jan. 2008, pp. 60-68, doi:10.1021/ar700089b

[12] J. Yeha, Y. B. Ling, and J. M. Karpd, et al., Micromolding of shape-controlled, harvestable cell-laden hydrogels," Biomaterials, Nov.2006, Vol.27, pp. 5391-5398, doi:10.1016/ j.biomaterials.2006.06.005.

[13] W. J. Jeong, J. Y. Kim and J. Choo, et al., -Continuous Fabrication of Biocatalyst Immobilized Microparticles Using Photopolymerization and Immiscible Liquids in Microfluidic Systems," Langmuir, Vol. 21, Mar. 2005, pp. 3738-3741, Doi:10.1021/la0501051. 